\title{
Targeted transcriptional repression using a chimeric TALE-SRDX repressor protein
}

\author{
Magdy M. Mahfouz • Lixin Li • Marek Piatek • \\ Xiaoyun Fang • Hicham Mansour • Dhinoth K. Bangarusamy • \\ Jian-Kang Zhu
}

Received: 27 October 2011/Accepted: 27 November 2011/Published online: 14 December 2011

(C) The Author(s) 2011. This article is published with open access at Springerlink.com

\begin{abstract}
Transcriptional activator-like effectors (TALEs) are proteins secreted by Xanthomonas bacteria when they infect plants. TALEs contain a modular DNA binding domain that can be easily engineered to bind any sequence of interest, and have been used to provide user-selected DNAbinding modules to generate chimeric nucleases and transcriptional activators in mammalian cells and plants. Here we report the use of TALEs to generate chimeric sequencespecific transcriptional repressors. The dHax3 TALE was used as a scaffold to provide a DNA-binding module fused to the EAR-repression domain (SRDX) to generate a chimeric repressor that targets the $R D 29 A$ promoter. The $\mathrm{dHax} 3$.SRDX protein efficiently repressed the transcription of the RD29A::LUC transgene and endogenous $R D 29 A$ gene in Arabidopsis. Genome wide expression profiling showed that the chimeric repressor also inhibited the expression of several other genes that contain the designer TALE-target sequence in their promoters. Our data suggest that TALEs
\end{abstract}

Electronic supplementary material The online version of this article (doi:10.1007/s11103-011-9866-x) contains supplementary material, which is available to authorized users.

M. M. Mahfouz $(\bowtie) \cdot$ L. Li $\cdot$ M. Piatek $\cdot$ X. Fang

Center for Plant Stress Genomics and Technology, King

Abdullah University of Science and Technology, Thuwal 23955-

6900, Kingdom of Saudi Arabia

e-mail: magdy.mahfouz@kaust.edu.sa

H. Mansour · D. K. Bangarusamy

BioScience Core Laboratory, King Abdullah University of Science and Technology, Thuwal 23955-6900, Kingdom of Saudi Arabia

\section{J.-K. Zhu (}

Department of Horticulture and Landscape Architecture, Purdue University, West Lafayette, IN 47907, USA

e-mail: jkzhu@purdue.edu can be used to generate chimeric repressors to specifically repress the transcription of genes of interest in plants. This sequence-specific transcriptional repression by direct on promoter effector technology is a powerful tool for functional genomics studies and biotechnological applications.

Keywords Chimeric repressors - TALE-based repressors $\cdot$ Targeted repression $\cdot$ EAR-repression domain

\section{Introduction}

Plants are sessile organisms and rely on a wide array of molecular mechanisms to control and adjust their adaptive responses to developmental and environmental cues. Understanding these responses requires that we understand the functioning of plant genes. Although such understanding has become increasingly possible with rapid advances in DNA sequencing technologies, the challenge is to turn information about gene sequence into knowledge about gene function. The analysis of the completed plant genome sequences revealed that transcriptional regulation plays a very pronounced role in plants (Riechmann et al. 2000). The Arabidopsis genome is predicted to contain 25,498 genes, and more than 2000 of these are transcription factors. Consequently, the percentage of transcription factor genes relative to the whole gene content is higher in Arabidopsis (6-10\%) than in organisms with genomes of similar size including Drosophila melanogaster (4.7\%) and Caenorhabditis elegans (3.6\%) (Riechmann et al. 2000). Plants use many different transcription factors to modulate gene expression and achieve the correct spaciotemporal control of gene activation and repression. Researchers use factors that activate or repress gene expression to understand gene function and phenotypic effects (Krogan and 
Long 2009). Transcriptional repression effects occur at several levels and involve active and passive repressors. Active repressors exhibit an intrinsic repression activity because they have defined repression domains that can interfere with the formation of the transcription pre-initiation complex and basal transcription (Krogan and Long 2009; Mahfouz 2010). Passive repressors, in contrast, interfere with transcriptional activators and prevent their binding to the DNA to cause transcription repression (Kazan 2006; Krogan and Long 2009).

Chimeric repressors, which have been used to study gene function in animal and plant systems, have a DNAbinding domain or a transcription factor fused to a variety of repression domains (Beerli et al. 2000; de Haan et al. 2000; Ohta et al. 2001). Transcription repressors that contain the EAR-repression domain (ERF-associated amphiphilic repression) were the first to be identified in plants (Kagale and Rozwadowski 2011; Ohta et al. 2001). The exact mechanism of function of the transcriptional repressors is not known but may involve interaction with co-repressors (Szemenyei et al. 2008). Researchers have fused the EAR motif to a number of transcription factors to generate dominant chimeric repressors (Hiratsu et al. 2003; Ikeda and Ohme-Takagi 2009; Matsui et al. 2008; Mito et al. 2010; Tsutsui et al. 2009). The application of this technology, however, is limited to the pre-existing transcription factors. In the chimeric repressor silencing technology or "CRES-T" system, the transcription factors were used as a DNA-binding module to repress the transcription factor's target genes but because many of the transcription factor targets are not known, it is difficult or impossible to achieve repression to specific genes. This repression technology would be much more powerful if it could be applied to a user-selected and defined single locus or multiple loci.

A chimeric transcription repressor or activator, in principle, is composed of a DNA-binding domain, an activation or repression domain, and a nuclear-localization signal (Guan et al. 2002; Yaghmai and Cutting 2002). Exploiting this fundamental principle to generate chimeric repressors or activators with genome-wide applicability requires a DNA-binding domain that can be modified and adapted to selectively and specifically bind to any DNA sequence in the genome. This kind of DNA-binding domain exists in phytopathogenic bacteria in the genus Xanthomonas. Phytopathogenic Xanthomonas spp. use the type III secretion system to inject transcription activator-like effectors (TALEs) into plant cells (Boch and Bonas 2010). These TALEs, which act as virulence factors, translocate to the nucleus and function as transcription factors by binding to their DNA targets in the promoter regions and reprograming the gene expression of the host (Kay et al. 2007; Romer et al. 2007). The repeats of the DNA-binding domains (DBD) dictate the specificity of TALEs to their
DNA target (Boch et al. 2009; Moscou and Bogdanove 2009). The repeats are nearly identical, and each repeat is composed of 34 or 35 amino acids and has a repeat variable diresidue (RVD) at positions 12 and 13. There is a one-toone correspondence between each RVD and a single nucleotide in the DNA target, and hence the identity and the sequence of the RVDs determine the specificity of each TALE to its target (Boch et al. 2009; Moscou and Bogdanove 2009).

The Brassicaceae pathogen $X$. campestris pv. armoraciae strain 5 produces Hax3, which is a member of the AvrBs3 family of TALEs (Kay et al. 2005). Hax3 TALE protein has all the structural features of TALEs including a DNA-binding domain composed of 11.5 repeat units, a nuclear-localization signal, and an acidic activation domain. The chief advantage of TALEs for the design of transcriptional repressors or activators is their versatility; they could be used, in principle, to target any functional domain to any gene locus by fusing the functional domain to an appropriately modified TALE DBD (Christian et al. 2010; Li et al. 2011a; Mahfouz et al. 2011; Morbitzer et al. 2010; Weber et al. 2011). Thus, TALEs could be used to take advantage of the entire repertoire of the transcriptionregulatory domains to achieve the desired level and type of regulation for any gene of interest. Hax3 recognizes a 12-bp DNA-binding element and has been used to generate a de novo engineered hybrid nuclease capable of generating double-strand breaks in vitro and in vivo in plants (Mahfouz et al. 2011).

Here, we report the generation of a chimeric transcriptional repressor that targets an element in the RD29A promoter. Our data demonstrate that the dHax3.SRDX chimeric repressor selectively suppresses the expression of the $R D 29 A:: L U C$ transgene and the $R D 29 A$ endogenous gene as well as several other genes with the target element in their promoters in Arabidopsis. This gene repression technology will be useful for functional genomics studies in crops and should have significant biotechnological applications.

\section{Results}

Design and generation of the $d$ Hax3.SRDX TALEbased transcriptional repressor to target an $R D 29 A$ promoter element

To generate a chimeric TALE-based repressor, we selected the Hax3 TALE as a scaffold and the SRDX repression domain; the Hax3 TALE provided a precise and selective DNA-binding module while the SRDX repression domain (Heyl et al. 2008) provided transcriptional repression. The natural Hax3 TALE binds to a 12-bp DNA box that 
contains the TACACCCAAACAT nucleotide sequence. The 11.5 repeats of $\mathrm{dHax} 3$ were modified to bind a 12-bp sequence (TCCCTTTATCTCT) in the RD29A promoter; this sequence overlaps by two nucleotides with the abscisic acidresponsive element (ABRE) (Fig. 1a) (Mundy et al. 1990). The $\mathrm{dHax} 3$ recognition box is preceded by the T nucleotide, 13-bp binding box, which was shown to be essential for TALEs binding to their targets (Boch et al. 2009). To generate the hybrid $d$ Hax3.SRDX repressor, we fused in frame the nucleotide sequence corresponding to 12 aa of the SRDX dominant repressor (LDLDLELRLGFA) to the full-length dHax3 sequence (Fig. 1b) (Hiratsu et al. 2003; Matsui and Ohme-Takagi 2010). As we previously reported, the $d \operatorname{Hax} 3$ fragments were assembled and fused to generate the fulllength $d \operatorname{Hax} 3$ (Mahfouz et al. 2011), which was then fused at its C-terminus to a fragment containing the SRDX domain. The dHax3.SRDX sequence was cloned in the pENTR/D gateway vector, and the construct was confirmed by sequencing. The in silico-designed chimeric repressor, herein referred to as dHax3.SRDX, has 972 aa residues and an estimated molecular mass of $102.77 \mathrm{kDa}$. The dHax3.SRDX was subsequently sub-cloned in the pET32a gateway compatible protein expression vector by LR reaction to generate thioredoxin.6His fusion protein (Trx.His.dHax3.SRDX). The bacterially expressed Trx.6His.dHax3.SRDX was shown to migrate in SDS polyacrylamide gel electrophoresis according to its expected size (Fig. 2a).

Hax3.SRDX chimeric repressor protein binds to its target sequence in vitro

To examine the ability of the bacterially expressed dHax3.SRDX chimeric repressor to bind to its DNA target,
(A)

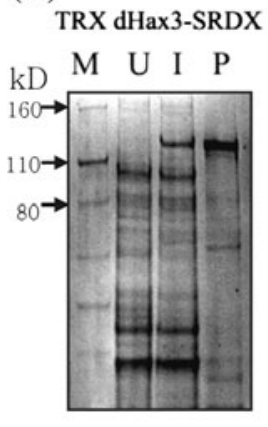

(B)

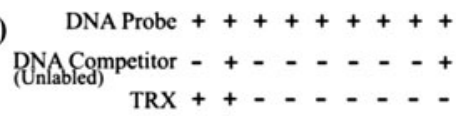

TRX dHax3-SRDX - - -

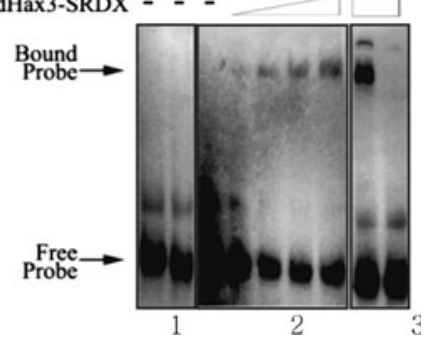

Fig. 2 Purification of dHax3.SRDX and EMSA. a Bacterial expression and purification of $\mathrm{dHax} 3$.SRDX protein. Lane 1 shows the protein marker, lane 2 shows the un-induced cell extract, lane 3 shows the IPTG-induced cell extract, and lane 4 shows the purified dHax3.SRDX fraction. b EMSA of dHax3.SRDX binding to its target sequence. Panel 1: the thioredoxin tag does not bind to the biotinlabeled probe. Panel 2: the dHax3.SRDX protein binds to the biotinlabeled probe sequence in a concentrationdependent manner. Panel 3: the unlabeled probe competitively reduces the binding of the biotinlabeled probe to the dHax3.SRDX protein

we performed electrophoretic mobility shift assays (EMSA) using the bacterially expressed dHax3.SRDX and a biotin-labeled double-stranded oligo-nucleotide containing the 12-bp DNA target sequence preceded by the T nucleotide. The assays showed that the dHax3.SRDX was capable of binding to its target DNA sequence and that the binding was competitively reduced by the addition of the same but unlabeled double-stranded oligo-nucleotides. The results suggest that the fusion of the SRDX domain did not affect the ability of the dHax3.SRDX chimeric repressor protein to bind to its target site (Fig. 2b).

Fig. 1 Sequence of $R D 29 A$ promoter showing effectorbinding elements and schematic diagram of dHax3.SRDX. a The sequence of the RD29A promoter showing the dHax3.SRDX-binding element (EBE), the dehydrationresponsive element (DRE), and the $\mathrm{ABA}$ responsive element (ABRE). b Structural representation of dHax3.SRDX showing the $\mathrm{N}$-terminal domain, repeat domain, a linker domain, a nuclear-localization signal, acidic activation domain, and the SRDX domain with its amino acid sequence shown below. Also shown below are the repeat variable diresidues (RVDs) of dHax3.SRDX with the sequence of its DNAbinding element (EBE)
(A)

-251 DRE

IACTACCGACATGAGTTCCAAAAAGCAAAAAAAAAGATCAAGCCGACACAGACACGCGTAGA ABRE EBE

GAGCAAAATGACTTTGACGTCACACCACGAAAACAGACGCTTCATACGTGTCCCTTTATCTCTC $+1$ .....TATA.....'ATG...'UUC

(B) dHax3.SRDX

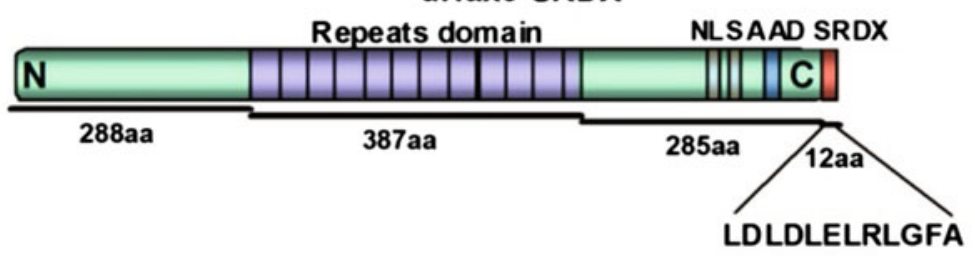

$\begin{array}{lllllllllllll}0 & 1 & 2 & 3 & 4 & 5 & 6 & 7 & 8 & 9 & 10 & 11 & 11.5\end{array}$

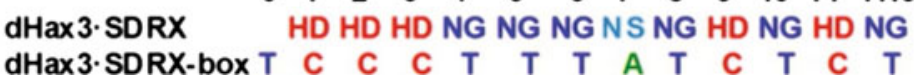


Expression of $d$ Hax3.SRDX repressor results in repression of the $R D 29 A$ promoter in vivo

To test whether the dHax3.SRDX causes transcriptional repression of the luciferase transgene driven by the $R D 29 A$ promoter and the endogenous RD29A gene in their chromosomal contexts, we generated stable transgenic lines of Arabidopsis ectopically expressing Hax3.SRDX under the cauliflower mosaic virus $35 \mathrm{~S}$ promoter in a background containing the RD29A::LUC transgene (ecotype C24, referred to as the WT). Several transgenic lines overexpressing 35S::dHAX3.SRDX were selected on MS nutrient medium supplemented with hygromycin at $30 \mathrm{mg} /$ L. Homozygous T3 plants expressing 35S::dHAX3.SRDX were used to analyze the transcriptional repression of the $R D 29 A$ promoter in both the luciferase transgene and the endogenous $R D 29 A$ gene.

$R D 29 A$ is one of the most well studied promoters (Yamaguchi-Shinozaki and Shinozaki 1994). This promoter contains the $\mathrm{ABRE}$ and dehydration responsive element (DRE) (Narusaka et al. 2003). The promoter can be activated by osmotic and cold stresses as well as by ABA(Ishitani et al. 1997). Because the DRE element can be activated by osmotic and cold stresses but not by ABA, this promoter has been used to analyze ABA-dependent and ABA-independent signaling (Ishitani et al. 1997; Xiong et al. 1999a). The DNA-binding box of the dHax3.SRDX hybrid repressor overlaps with $2 \mathrm{bp}$ of the ABRE in the RD29A promoter and no overlap with the DRE box (Fig. 1a). We tested the transcriptional repression of the $R D 29 A$ promoter by single or combined treatments of Arabidopsis plants expressing dHax3.SRDX with ABA and cold. Six lines of Arabidopsis over-expressing dHax3.SRDX showed significant repression of the endogenous RD29A gene and the luciferase transgene (Fig. 3a, b). The treatment of WT with $100 \mu \mathrm{m}$ ABA resulted in the activation of the RD29A promoter and strong luminescence as shown in Fig. 3a. In contrast to the WT, ectopic expression of the $d$ Hax3.SRDX led to the suppression of the $R D 29 A$ promoter, resulting in minimal or no luminescence. Our data show that the dHax3.SRDX strongly represses the $R D 29 A$ promoter.

Because the $R D 29 A$ promoter is also induced by salt, we tested the ability of the dHax3.SRDX chimeric repressor to repress the $R D 29 A$ promoter after salt treatment $(300 \mathrm{mM}$ $\mathrm{NaCl}, 3 \mathrm{~h}$ ). Our data show that the dHax3.SRDX chimeric repressor can significantly repress the $R D 29 A$ promoter after salt treatment (Fig. 3c).

It was previously reported that low temperature and ABA treatments have additive effects on the transcriptional induction of the RD29A::LUC transgene and the RD29A endogene (Xiong et al. 1999b). Because cold and ABA activate the $R D 29 A$ promoter via two different signaling pathways, we examined the ability of the dHax3.SRDX chimeric repressor to simultaneously repress gene transcription activated by both signaling pathways. We performed a combined treatment of cold ( $48 \mathrm{~h}$ at $4 \mathrm{C}^{\circ}$ ) and ABA $(100 \mu \mathrm{m}$ for $3 \mathrm{~h})$ on the same dHax3.SRDX lines. Our data indicate that the designer chimeric repressor is capable of repressing the $L U C$ expression in response to two independent signaling pathways (Fig. 4a).

Since the repression might be mediated merely by the binding of the dHax3 effector, to its promoter, and not by SRDX repression domain of $\mathrm{dHax} 3$.SRDX repressor, we tested this possibility by functional analysis in $N$. benthamiana transient assays. We inserted the dHax3.SRDX effector-binding element in the $B s 3$ minimal promoter to generate $\Delta \mathrm{d} H a x 3 B s 3::$ uidA and co-delivered this construct or its WT version (Bs3::uidA) with $35 \mathrm{~S}:: \mathrm{dHax} 3$ or 35S::dHax3.SRDX, respectively (Morbitzer et al. 2011). Our data clearly indicate that the dHax3 activates the $\Delta \mathrm{d} H a x 3 B s 3::$ uidA expression (Fig. 4b, panel 2). dHax3.SRDX, however, strongly repressed the expression

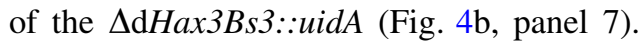

To confirm the luminescence data at the molecular level, we analyzed the abundance of the luciferase and $R D 29 A$ transcripts using real-time RT-PCR. The endogenous $R D 29 A$ gene and RD29A::LUC transgene were induced and highly expressed in the WT control plants treated with $100 \mu \mathrm{m}$ ABA for $3 \mathrm{~h}$ (Fig. 5a, b). The dHax3.SRDX chimeric repressor lines, however, showed substantially lower levels of the $R D 29 A$ and $L U C$ transcripts, indicating that their expression was repressed. These data are consistent with the bioluminescence data and indicate that the dHax3.SRDX protein functions as a strong chimeric repressor in planta.

\section{Genome-wide effect of the dHax3.SRDX chimeric} repressor on gene expression

Several reports have shown that TALEs can be used as DNA-binding modules with high specificity and precision (Li et al. 2011a; Morbitzer et al. 2010; Zhang et al. 2011). TALEs with a higher number of repeats and longer DNA targets might be more specific than those with shorter repeats and DNA targets (Boch et al. 2009). To test the specificity of the dHax3.SRDX chimeric repressor, we used the Patmatch algorithm (http://is.gd/g31n9) to scan the Arabidopsis genome for sequences that contain matching boxes specifically in the promoter regions. We identified in the $-500 \mathrm{bp}$ upstream sequence only one perfect match that corresponds to the RD29A promoter (AT5G52310). The code of the RVDs DNA-binding specificities, however, is somewhat degenerate. For example, the NS RVD could bind to A, C, G, or T nucleotide, with higher affinity to $\mathrm{A}$ and reduced affinity to $\mathrm{C}, \mathrm{G}$, and $\mathrm{T}$ nucleotides (Boch et al. 2009; Miller et al. 2011). We scanned the Arabidopsis 
Fig. 3 Transcriptional

repression of $R D 29 A: \because L U C$ in

dHax3.SRDX transgenic plants. a WT (C24 RD29A::LUC) and dHax3.SRDX expressing lines $1,2,5$, and 6 grown on MS agar plates for 1 week were treated with $100 \mu \mathrm{m} \mathrm{ABA}$ for $3 \mathrm{~h}$. Luminescence images were taken after the ABA treatment (left). The right panel shows the label details of dHax3.SRDX expressing lines. b Two-week old plants from the same lines as (a) were treated with cold $\left(4^{\circ} \mathrm{C}\right)$ for $48 \mathrm{~h}$. Luminescence images were taken after the cold treatment (left)

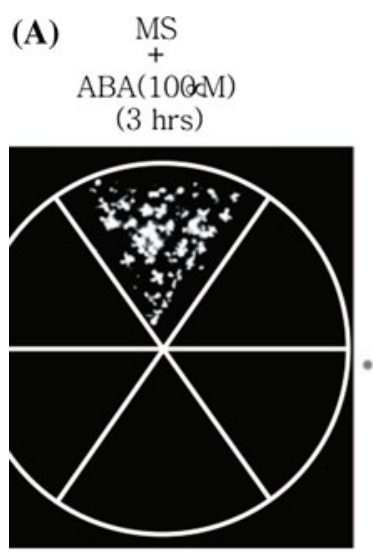

Luminescence

(B)

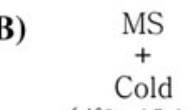

$\left(4^{\circ} \mathrm{C}, 48 \mathrm{hrs}\right)$

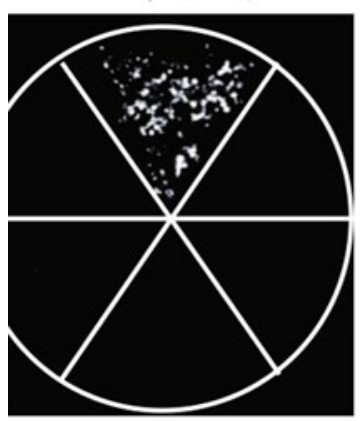

Luminescence

(C)
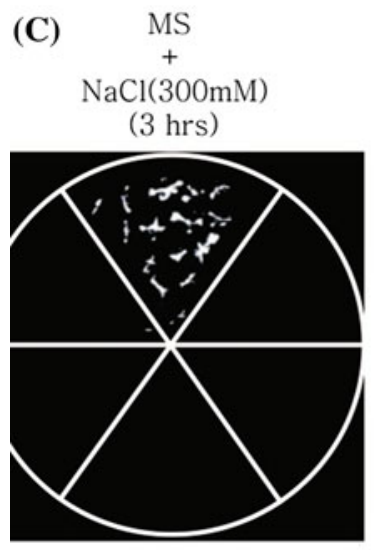

Luminescence
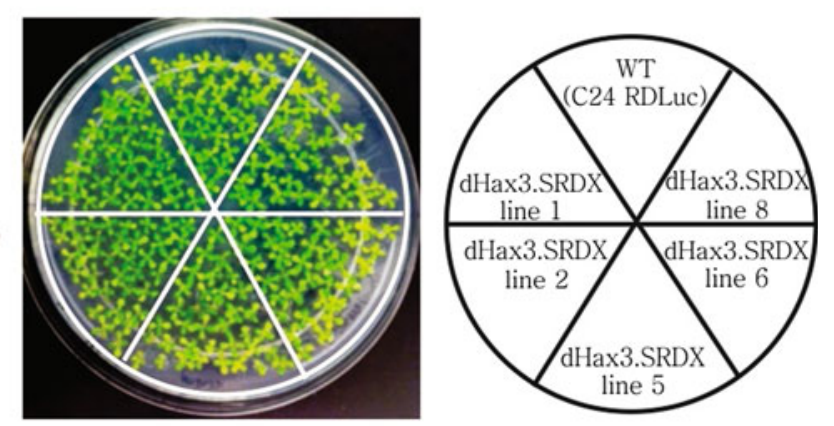

White Field

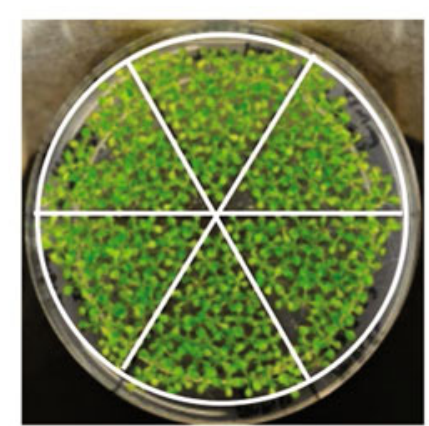

White Field

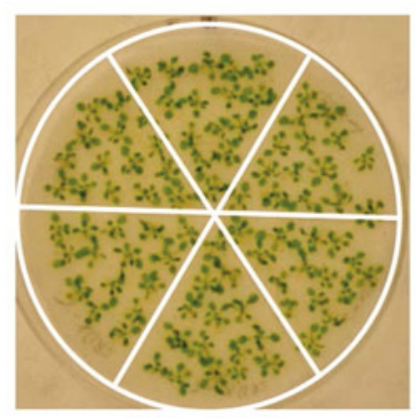

White Field genome for potential dHax3.SRDX binding to DNA boxes that include TCCCTTTATCTCT, TCCCTTTCTCTCT, TC CCTTTGTCTCT, or TCCCTTTTTCTCT in the $-500 \mathrm{bp}$ upstream promoter region. We found one perfect match, in the RD29A gene promoter that includes the TCCCT TTATCTCT box, and 20 other possible binding hits on either strand, including $\mathrm{C}, \mathrm{G}$, or T nucleotide corresponding to the 7 th repeat in the tandem repeat region of the DNA-binding module. Moreover, we identified 70 additional possible targets when we extended the search to $-3,000 \mathrm{bp}$ upstream promoter regions and 82 binding hits in the genes including introns and UTR regions.

To examine the selectivity and specificity of the dHax3.SRDX repressor, we studied the expression patterns of these genes in the $d \operatorname{Hax} 3 . S R D X$ transgenic lines. We performed a microarray experiment to investigate the transcript levels of these potential targets in the WT control and the dHax3.SRDX lines. We found that the gene hits in 
(A)

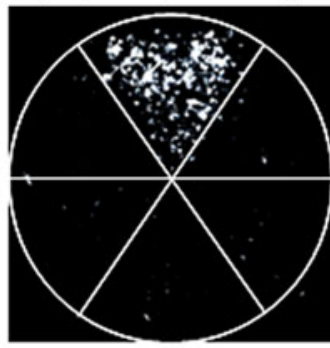

Luminescence

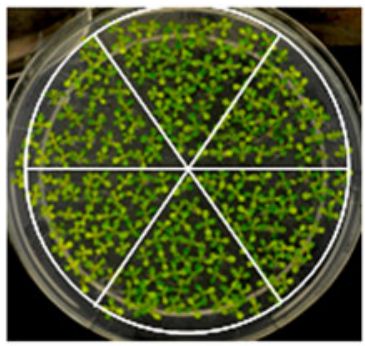

White Field

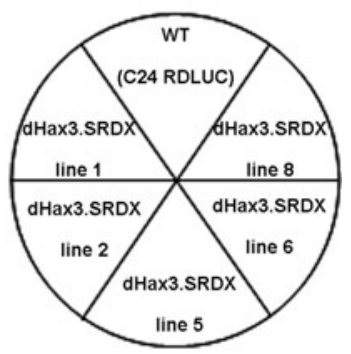

(B)

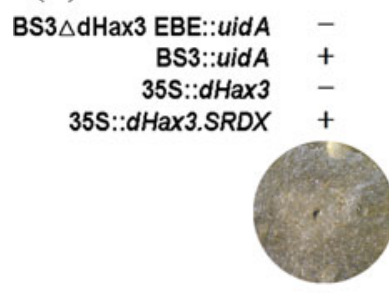

1

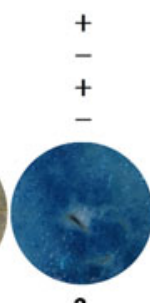

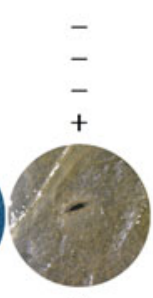

3
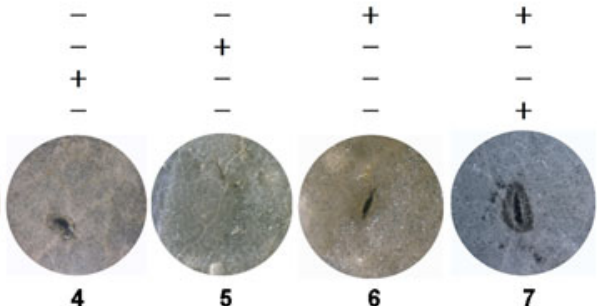

7
Fig. 4 The ability of dHax3.SRDX to repress $R D 29 A: \because L U C$ after a combined treatment with $\mathrm{ABA}$ and cold and the transcriptional repression is due to the presence of the SRDX domain. a One-weekold plants grown on MS agar plates were treated with cold $\left(4^{\circ} \mathrm{C}, 48 \mathrm{~h}\right)$ followed by ABA $(100 \mu \mathrm{m}, 3 \mathrm{~h})$. Luminescence images were taken after the combined treatments. All dHax3.SRDX expressing lines showed a significant repression of $R D 29 A:: L U C$ after the combined

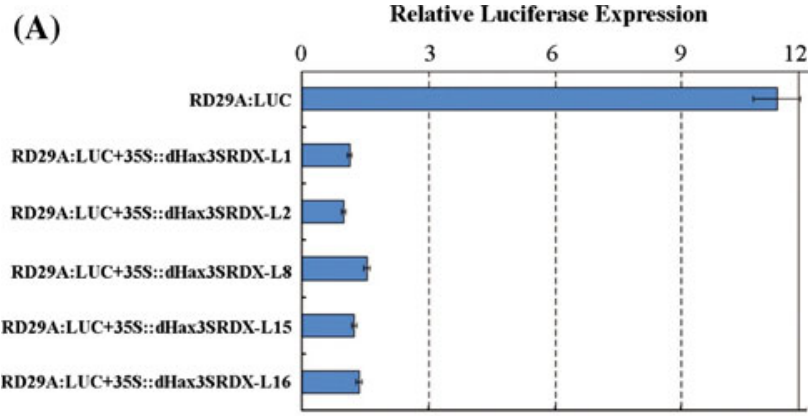

(B)

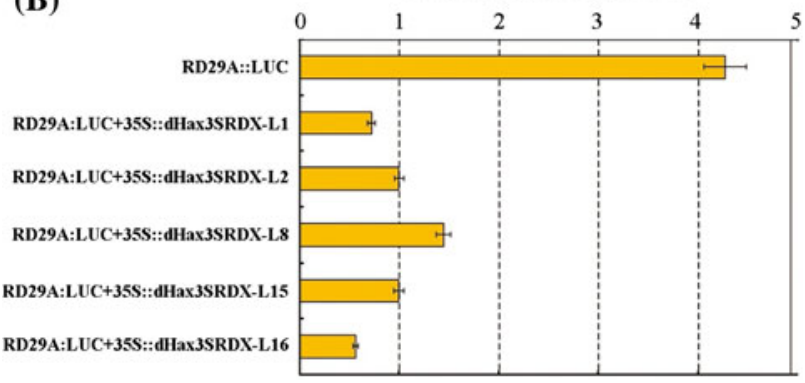

Fig. 5 Q-PCR analysis of RNA extracted from ABA-treated C24 RD29A:: LUC WT control and dHax3.SRDX expressing lines. Experiments were normalized relative to actin2, and two or more biological replicates were tested with similar results treatments. WT (control) luminescence indicates the transcriptional activities of the RD29A::LUC promoter. b uidA repression is mediated by the SRDX repression domain. In planta functional analysis of dHax3 activator and dHax3.SRDX chimeric repressor. The constructs uidA driven by $B s 3$ WT or $B s 3 \Delta d H a x 3$ promoters were co-delivered via Agrobacterium tumefaciens into N. benthamiana leaves and the GUS assays were performd $48 \mathrm{hpi}$

the $-500 \mathrm{bp}$ upstream promoter regions were underexpressed by at least twofold across all dHax3.SRDX lines. The repressed genes that contain the binding box (ATX1, AT2G07280, AT5G08390, AAP6, AT2G41230, ATTAP1, AT5G45580, LTI78) are shown in blue boxes and their relative distance from the transcriptional start is shown at left and the nucleotides that bind to the NS 7th repeat are shown at the right (Fig. 6). The distance from the transcriptional start seems to be important since in most of the repressed genes the target sequence lies within the $-500 \mathrm{bp}$ of the promoter region. Interestingly, in most of the lines we found $>20$-fold repression of two gene hits (AT2G41230 and AT2G07280), which have the target sequence within $-3,000$ bp upstream promoter regions. The strong repression of these two genes might indicate the importance of the chromatin environment, the distance between the binding sequence and transcriptional start or the presence of multiple potential binding sequences that may lead to the recruitment of more repressor proteins or tighter binding. We also noticed that one predicted target (AAP6) was over-expressed by twofold (Fig. 6). The over-expression of this gene hit might be an indirect consequence of the repression of one or more of the repressed targets. Importantly, we did not find transcriptional modulation in genes that do not have the target 


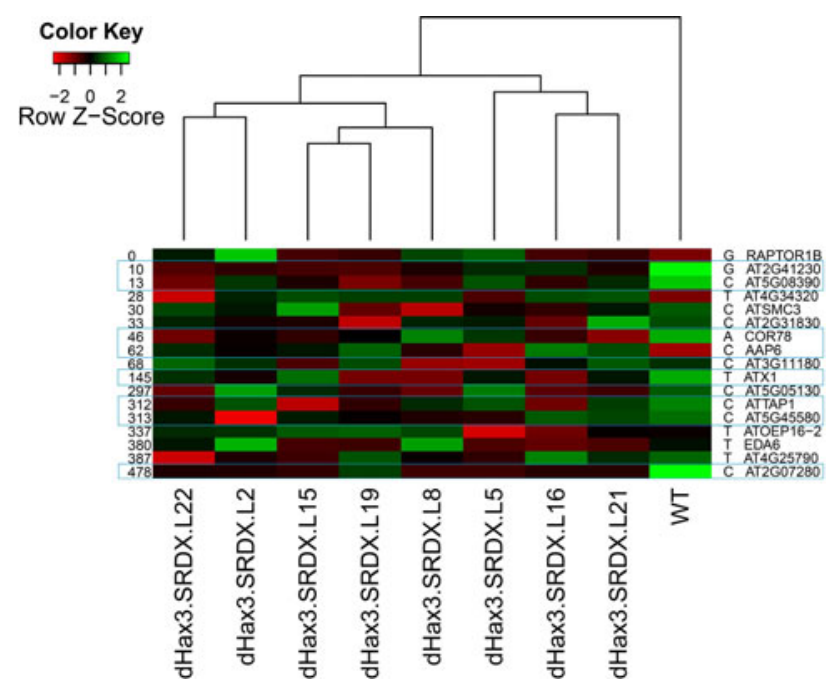

Fig. 6 Microarray expression analysis of dHax3.SRDX over-expression lines showing the repression of target genes containing the TCCCTTT[A/C/G/T]TCTCT DNA-binding box within the promoter region. Samples (columns) were clustered using Euclidean distance and complete linkage. Genes (rows) with motif variations were sorted in ascending order according to the distance to the $5^{\prime}$ UTR start. Genes in the blue frames (discussed in text) compared to the WT control are showing transcriptional repression across all tested dHax3.SRDX lines

sequence in their promoters. Our data demonstrate that the dHax3.SRDX chimeric repressor inhibits the expression of genes with the designed target sequences in their promoters, and suggest that the off-target effects can be predicted and avoided in the design of such chimeric repressors.

\section{Discussion}

In the present study, we demonstrate that the TALE-based dHax3.SRDX chimeric protein designed to target the $R D 29 A$ promoter can repress the RD29A::LUC transgene and $R D 29 A$ endogenous gene in a chromosomal context and thus can be used as an effective artificial repressor. The repeats of the dHax3 DNA-binding domain were modified so that it can bind to a DNA box in the RD29A promoter. The dHax3 was fused in frame to the SRDX domain to generate the $d H a x 3 . S R D X$. Our data suggest that the fusion of the SRDX domain does not affect the conformation of the $\mathrm{dHax} 3$ since the fusion protein was capable of binding to its target sequence in vitro (Fig. 2b). dHax3.SRDX, driven by the CaMV $35 \mathrm{~S}$ promoter, was ectopically expressed in Arabidopsis containing the RD29A::LUC transgene. The $R D 29 A$ promoter can be activated by a variety of environmental stresses including salt, drought, cold, and also the phytohormone ABA (Chinnusamy et al. 2002; Hua et al. 2006; Ishitani et al. 1997; Kasuga et al. 2004). To test the ability of the dHax3.SRDX to repress its target genes, we used luciferase imaging to analyze $L U C$ expression in several Arabidopsis lines expressing dHax3.SRDX. Arabidopsis dHax3.SRDX lines grown on MS media and treated with $100 \mu \mathrm{m}$ ABA for $3 \mathrm{~h}$ showed a significant suppression of the luciferase signal relative to the wild type control, indicating the repression of the RD29A::LUC transgene by the dHax3.SRDX chimeric repressor protein (Fig. 3a). The dHax3.SRDX also suppressed the expression of $R D 29 A:: L U C$ transgene in response to cold or salt treatments (Fig. 3b, c). Moreover, our data demonstrate the ability of the dHax3.SRDX to suppress the expression of the RD29A::LUC transgene after a combined $\mathrm{ABA}$ and cold treatment, indicating its ability to simultaneously inhibit the activities of multiple transcriptional activators, which integrate signals from different signaling pathways (Fig. 4).

Our data indicate that the dHax3.SRDX chimeric repressor protein suppressed the transcription of a RD29A::LUC transgene and RD29A endogenous gene in a chromatin context. These data demonstrate the versatility of the repression mechanism and the ability of the TALEbased repressors to suppress the expression of more than one gene simultaneously. It should be noted that the transient and stable expression of TALE protein alone did not suppress the expression of RD29A promoter indicating that the repression is due to the presence of the SRDX repression domain (Fig. 4b) (Mahfouz et al. 2011). Moreover, the use of the strong $35 \mathrm{~S}$ promoter might not be necessary to obtain gene repression effects. A lower ratio of repressor to activator was able to cause efficient suppression of the reporter gene in Arabidopsis transient assays (Fujimoto et al. 2000). Our data demonstrate a significant reduction in the expression of the endogenous $R D 29 A$ gene, although the repression of $R D 29 A$ was generally less than that of the RD29A::LUC transgene as evidenced by real-time RT-PCR (Fig. 5a, b). The ability of the TALE repressor to bind to its target might be affected by the chromatin status or the distance of the TALE binding motif from the transcriptional start site may influence its repression activity.

Because the dHax3.SRDX chimeric repressor contains the NS-type RVD at the 7th position, we tested the ability of the repressor to repress genes that contain binding boxes where the 7th nucleotide in the DNA target is A, C, G, or T. We identified 21 potential hits in the -500 bp upstream promoter region and 70 more gene hits in the $-3,000 \mathrm{bp}$ upstream region. It should be mentioned that these are not off-targets since they have the TALE-SRDX DNA binding box according to the TALE DNA binding code. Off-targets are defined as the genes whose level of expression is modulated due to the expression of TALE-SRDX and may contain a non-perfect match of the DNA binding box. Our microarray data indicate that the expression of 5 gene hits in the -500 bp regions is repressed in all tested lines. 
The repression levels vary among the targets perhaps because of the chromosomal position effect or the distance from the transcription start site. Moreover, two gene hits (AT2G41230 and AT2G07280) containing the dHax3.SRDX DNA-binding box on the opposite strand in $-3,000$ bp upstream region, show significantly higher levels of repression. These two genes contain multiple repeats with similar sequence to the DNA-binding box TCCCTTT[ACGT]TCTCT within $-5 \mathrm{~kb}$ upstream of the transcription start site. In fact, there are eight repeats of 50-bp motifs with a DNA sequence similar to that of the DNA binding box (TCCCTTGTAAATCATTCT). The high level of repression of these two genes could be due to their local chromatin environments, the presence of the DNA-binding box on the opposite strand, the distance of the DNA-binding box relative to the transcriptional start site, or the presence of multiple copies of a motif similar to the DNA-binding box. The presence of multiple sequences similar to the DNA-binding box might help recruit a large number of the chimeric repressor to result in strong transcriptional repression. It appears that the transcriptional repression occurred regardless of the dHax3.SRDX binding orientation because the presence of the EBE on the forward or reverse strand relative to the gene resulted in repression activities. This is consistent with proposed models of SRDX-mediated repression where SRDX might function by disrupting the formation of the transcription complex or recruiting repressors and co-repressors. These data indicate that the chromatic status may affect the binding of the dHax3.SRDX or the function of the SRDX domain. Moreover, many details are unknown concerning the nature of TALE binding, including the chromatin environments and optimal distance from the transcription start site. These details are of paramount importance for the future design of effective TALE-based activators or repressors.

Because de novo TALEs (dTALEs) with higher repeat units might be expected to possess higher target specificity (Morbitzer et al. 2011), dHax3.SRDX chimeric repressors should be designed with at least 18 repeat units if a single gene is to be targeted. However, designing dTALEs with variable repeat lengths and RVDs with degenerate nucleotide binding would be useful for analyzing the nature and details of the binding and activity of dTALE activators and repressors. Several parameters for TALE target designs were suggested based on the study of positional biases, neighbor effects, and the binding of RVDs to nucleotides in the overall target (Cermak et al. 2011). According to these suggestions, there is strong bias against $\mathrm{T}$ at position $1, \mathrm{~A}$ at position 2 , and $\mathrm{G}$ at position 3 and the next to the last position, and a moderate bias for $\mathrm{T}$ at the last position (Cermak et al. 2011). These and other suggestions may be useful for designing effective TALE DNA-binding modules. Moreover, several TALEs repeats assembly protocols were recently reported, and these should facilitate the efficient design and generation of TALEs for user-selected targets (Cermak et al. 2011; Li et al. 2011b; Morbitzer et al. 2011; Zhang et al. 2011).

Our present study suggests that a single Hax3.SRDX protein can be designed to silence a single gene or a group of genes sharing DNA-binding elements in the promoter regions, making it possible to functionally characterize single genes, multiple genes or gene families. It remains to be determined whether the TALE-based repression technology could be a useful alternative to RNA interference (RNAi), a widely used technology that suffers from a variety of disadvantages and pitfalls. The demonstration of the effectiveness of the TALE-based repression technology to different promoters, gene families and chromosomal loci compared to RNAi is required to draw a conclusion. The primary disadvantage of RNAi is the off-target effects, i.e., sequence complementarities between an siRNA and the mRNA involving fewer than 10 nucleotides can lead to the reduction of expression (Birmingham et al. 2006). dTALE repressors, in contrast, can be designed to bind specifically to one genomic target. RNAi-based silencing exhibits variable effects depending on the target gene, the targeted region of the transcript, and even between different lines carrying the same RNAi construct (Wang et al. 2005). Moreover, RNAi phenotypes can be lost over several generations. Because RNAi suffers from problems related to efficacy, specificity and stability, silencing by TALE chimeric repressors might represent an attractive alternative with enormous potential for basic and applied research. For example, silencing by TALE chimeric repressors could be used for the genetic manipulation of commercially important crop plants (Mahfouz and Li 2011). This gene repression technology may also be applied to crop plants to identify genes responsible for traits of interest including biotic and abiotic stress resistance, nitrogen use efficiency, high yield, and control of fertility.

\section{Materials and methods}

\section{dHax3.SRDX design and vector construction}

pET32a.dHax3.SRDX was generated by performing LR recombination reactions between the dHax3.SRDX entry clone and the gateway-compatible pET32a expression vector according to manufacturer's protocol (Invitrogen). The expression clone was transformed into Escherichia coli BL21, and the protein expression was induced at $25^{\circ} \mathrm{C}$ for $5 \mathrm{~h}$ with $1 \mathrm{mM}$ isopropyl $\beta$-d-1thiogalactopyranoside (IPTG). The TRX.dHax3.SRDX protein was purified using Qiagen Ni-NTA agarose resin according to the manufacturer's instructions. LR reactions were performed between the 
pENTR/dHax3.SRDX entry clone and the pMDC32 gateway compatible binary vector to generate the final construct for in planta analysis. The pMDC32/dHax3.SRDX clone was confirmed by sequencing and transformed into Agrobacterium tumefaciens GV3101 and then to Arabidopsis plants by the floral dipping method (Zhang et al. 2006).

\section{Electrophoretic mobility shift assay (EMSA)}

EMSA was performed using the Lightshift Chemiluminescent kit (Pierce) and following the manufacturer's instructions. For dHax3.SRDX DNA-binding studies, the TRX.dHax3.SRDX protein was purified as described above, and the protein concentration was measured with a Bradford protein assay kit (Bio-Rad). Complementary pairs of $5^{\prime}$ biotin-labeled or non-labeled oligonucleotides were annealed and used as probes for the binding studies. EMSA binding-reaction buffer contained $12 \mathrm{mM}$ Tris-HCL, $60 \mathrm{mM} \mathrm{KCl}, 1 \mathrm{mM}$ DTT, 2.5\% glycerol, $5 \mathrm{mM} \mathrm{MgCl}_{2}$, $50 \mathrm{ng} / \mu \mathrm{L}$ poly(dI.dC), $0.05 \%$ Nonidet $\mathrm{P}-40,0.2 \mathrm{mM}$ EDTA, 35 fmol biotin-labeled DNA, 10 pmol unlabeled DNA, and 30-480 fmol TRX.His fusion proteins. The EMSA binding reactions were kept on ice for $10 \mathrm{~min}$ before the biotin-labeled probe was added. Gel electrophoresis was performed using $8 \%$ Tris/Borate/EDTA (TBE) native ready-made gels from Invitrogen. Blotting was performed on a positively charged nylon membrane, and the membrane was cross-linked using CL-1000 UV cross-linker for $30 \mathrm{~s}$.

\section{Agrobacterium and Arabidopsis transformation}

Arabidopsis thaliana (ecotype C24) expressing the chimeric $R D 29 A:: L U C$ reporter gene (referred to as the wild type or WT in this study) was used for the in planta analysis of the dHax3.SRDX repressor. pMDC32.dHax3. SRDX was transformed into Agrobacterium strain GV3101 by the freeze-and-thaw method. A transformed single colony of Agrobacterium GV3101 was used to transform Arabidopsis C24 RD29A::LUC plants as described previously (Zhang et al. 2006). Transformants were selected on MS agar plates supplemented with $30 \mathrm{mg} / \mathrm{L}$ of hygromycin B. T3 plants were used for the transcriptional repression analysis.

\section{Assay of RD29A::LUC expression}

WT (C24 RD29A::LUC) and dHax3.SRDX over-expression lines were grown on MS agar media for 1 week. Seedlings were sprayed with $100 \mu \mathrm{m}$ ABA for $3 \mathrm{~h}$ or kept at $4^{\circ} \mathrm{C}$ for $24 \mathrm{~h}$ (cold treatment) or incubated on filter papers soaked with $300 \mathrm{mM} \mathrm{NaCl}$ (salt treatment) (Chinnusamy et al. 2002). Plates with treated plants were sprayed with Luciferin at room temperature and kept in the dark for $5 \mathrm{~min}$ before they were placed in the dark chamber of the CCD camera (PIXIS 2048). Image quantification was carried out using the Winview software supplied by the manufacturer.

RNA isolation and quantitative real-time PCR

The expression levels for the LUC transgene and RD29A endogenous gene were analyzed in the treated and untreated WT and dHax3.SRDX lines. Total RNA was purified using the RNeasy kit (Qiagen SA). For the cDNA synthesis, $1 \mu \mathrm{g}$ of total RNA was used for the reverse transcription using reverse trancriptase (Life Technologies, Applied Bioscience).

All cDNAs were measured by real-time PCR. Quantitative PCR was performed in an ABI prism 7,900 using a SYBR Green. The $\beta$-actin gene served as the internal control for normalization of data. All experiments are done in at least duplicate.

Microarray analysis

The RNeasy plant mini kit (Qiagen) was used to extract total RNAs from 2-week-old WT and dHax3.SRDX overexpression lines that were grown on MS agar plates and treated with $100 \mu \mathrm{M}$ ABA. The total RNA samples were pretreated with RNase-free DNase I and cleaned using the Plant Total RNA isolation kit (Qiagen). Probe preparation, hybridization to the Arabidopsis ATH1 Gene expression arrays (Agilent Technologies), and subsequent processing steps were performed according to the manufacturer's procedures. Raw signals were extracted from the scanned images; the background was subtracted and the data were normalized using the Feature Extraction Software (10.7.1.1; Agilent technologies). These signals were then $\log 2$ transformed and subjected to percentile shift-based normalization and median-based baseline transformation using Genespring GX (Agilent Technologies). WT (C24 RD29A::LUC) plant values were used as the baseline to calculate the intensity ratio/fold changes of the dHax3.SRDX plant lines. False discovery rates (FDRs) were calculated by significance analysis of microarrays algorithm, and genes with fold change of at least 2 and a FDR-corrected $\mathrm{P}$ value lower than 0.05 were identified.

Acknowledgments We thank Thomas Lahaye for providing the Bs3::uidA binary construct. We also thank Jose M. Pardo and Ray Bressan for helpful discussions and members of the genome-engineering group at KAUST for their feedback and technical assistance.

Open Access This article is distributed under the terms of the Creative Commons Attribution Noncommercial License which permits any noncommercial use, distribution, and reproduction in any medium, provided the original author(s) and source are credited. 


\section{References}

Beerli RR, Dreier B, Barbas CF 3rd (2000) Positive and negative regulation of endogenous genes by designed transcription factors. Proc Natl Acad Sci USA 97:1495-1500

Birmingham A, Anderson EM, Reynolds A, Ilsley-Tyree D, Leake D, Fedorov Y, Baskerville S, Maksimova E, Robinson K, Karpilow J et al (2006) 3' UTR seed matches, but not overall identity, are associated with RNAi off-targets. Nat Methods 3:199-204

Boch J, Bonas U (2010) Xanthomonas AvrBs3 family-type III effectors: discovery and function. Annu Rev Phytopathol 48:419-436

Boch J, Scholze H, Schornack S, Landgraf A, Hahn S, Kay S, Lahaye T, Nickstadt A, Bonas U (2009) Breaking the code of DNA binding specificity of TAL-type III effectors. Science 326:1509-1512

Cermak T, Doyle EL, Christian M, Wang L, Zhang Y, Schmidt C, Baller JA, Somia NV, Bogdanove AJ, Voytas DF (2011) Efficient design and assembly of custom TALEN and other TAL effector-based constructs for DNA targeting. Nucleic Acids Res 39:e82. doi: 10.1093/nar/gkr218

Chinnusamy V, Stevenson B, Lee BH, Zhu JK (2002) Screening for gene regulation mutants by bioluminescence imaging. Sci STKE 36:pl10

Christian M, Cermak T, Doyle EL, Schmidt C, Zhang F, Hummel A, Bogdanove AJ, Voytas DF (2010) Targeting DNA double-strand breaks with TAL effector nucleases. Genetics 186:757-761

de Haan G, Chusacultanachai S, Mao C, Katzenellenbogen BS, Shapiro DJ (2000) Estrogen receptor-KRAB chimeras are potent ligand-dependent repressors of estrogen-regulated gene expression. J Biol Chem 275:13493-13501

Fujimoto SY, Ohta M, Usui A, Shinshi H, Ohme-Takagi M (2000) Arabidopsis ethylene-responsive element binding factors act as transcriptional activators or repressors of GCC box-mediated gene expression. Plant Cell 12:393-404

Guan X, Stege J, Kim M, Dahmani Z, Fan N, Heifetz P, Barbas CF 3rd, Briggs SP (2002) Heritable endogenous gene regulation in plants with designed polydactyl zinc finger transcription factors. Proc Natl Acad Sci USA 99:13296-13301

Heyl A, Ramireddy E, Brenner WG, Riefler M, Allemeersch J, Schmulling T (2008) The transcriptional repressor ARR1-SRDX suppresses pleiotropic cytokinin activities in Arabidopsis. Plant Physiol 147:1380-1395

Hiratsu K, Matsui K, Koyama T, Ohme-Takagi M (2003) Dominant repression of target genes by chimeric repressors that include the EAR motif, a repression domain, in Arabidopsis. Plant $\mathrm{J}$ 34:733-739

Hua ZM, Yang X, Fromm ME (2006) Activation of the NaCl- and drought-induced RD29A and RD29B promoters by constitutively active Arabidopsis MAPKK or MAPK proteins. Plant Cell Environ 29:1761-1770

Ikeda M, Ohme-Takagi M (2009) A novel group of transcriptional repressors in Arabidopsis. Plant Cell Physiol 50:970-975

Ishitani M, Xiong L, Stevenson B, Zhu JK (1997) Genetic analysis of osmotic and cold stress signal transduction in Arabidopsis: interactions and convergence of abscisic acid-dependent and abscisic acid-independent pathways. Plant Cell 9:1935-1949

Kagale S, Rozwadowski K (2011) EAR motif-mediated transcriptional repression in plants: an underlying mechanism for epigenetic regulation of gene expression. Epigenetics 6(2):141-146

Kasuga M, Miura S, Shinozaki K, Yamaguchi-Shinozaki K (2004) A combination of the Arabidopsis DREB1A gene and stressinducible rd29A promoter improved drought- and low-temperature stress tolerance in tobacco by gene transfer. Plant Cell Physiol 45:346-350
Kay S, Boch J, Bonas U (2005) Characterization of AvrBs3-like effectors from a Brassicaceae pathogen reveals virulence and avirulence activities and a protein with a novel repeat architecture. Mol Plant Microbe Interact 18:838-848

Kay S, Hahn S, Marois E, Hause G, Bonas U (2007) A bacterial effector acts as a plant transcription factor and induces a cell size regulator. Science 318:648-651

Kazan K (2006) Negative regulation of defence and stress genes by EAR-motif-containing repressors. Trends Plant Sci 11:109-112

Krogan NT, Long JA (2009) Why so repressed? Turning off transcription during plant growth and development. Curr Opin Plant Biol 12:628-636

Li T, Huang S, Jiang WZ, Wright D, Spalding MH, Weeks DP, Yang B (2011a) TAL nucleases (TALNs): hybrid proteins composed of TAL effectors and FokI DNA-cleavage domain. Nucleic Acids Res 39:359-372

Li T, Huang S, Zhao X, Wright DA, Carpenter S, Spalding MH, Weeks DP, Yang B (2011b). Modularly assembled designer TAL effector nucleases for targeted gene knockout and gene replacement in eukaryotes. Nucleic Acids Res 39:6315-6325

Mahfouz MM (2010) RNA-directed DNA methylation: mechanisms and functions. Plant Signal Behav 5:806-816

Mahfouz MM, Li L (2011) TALE nucleases and next generation GM crops. GM Crop 2:99-103

Mahfouz MM, Li L, Shamimuzzaman M, Wibowo A, Fang X, Zhu JK (2011) De novo-engineered transcription activator-like effector (TALE) hybrid nuclease with novel DNA binding specificity creates double-strand breaks. Proc Natl Acad Sci USA 108:2623-2628

Matsui K, Ohme-Takagi M (2010) Detection of protein-protein interactions in plants using the transrepressive activity of the EAR motif repression domain. Plant J 61:570-578

Matsui K, Umemura Y, Ohme-Takagi M (2008) AtMYBL2, a protein with a single MYB domain, acts as a negative regulator of anthocyanin biosynthesis in Arabidopsis. Plant J 55:954-967

Miller JC, Tan S, Qiao G, Barlow KA, Wang J, Xia DF, Meng X, Paschon DE, Leung E, Hinkley SJ et al (2011) A TALE nuclease architecture for efficient genome editing. Nat Biotechnol 29:143-148

Mito T, Seki M, Shinozaki K, Ohme-Takagi M, Matsui K (2010) Generation of chimeric repressors that confer salt tolerance in Arabidopsis and rice. Plant Biotechnol J 9:736-746

Morbitzer R, Romer P, Boch J, Lahaye T (2010) Regulation of selected genome loci using de novo-engineered transcription activator-like effector (TALE)-type transcription factors. Proc Natl Acad Sci USA 107:21617-21622

Morbitzer R, Elsaesser J, Hausner J, Lahaye T (2011) Assembly of custom TALE-type DNA binding domains by modular cloning. Nucleic Acids Res. doi: 10.1093/nar/gkr151

Moscou MJ, Bogdanove AJ (2009) A simple cipher governs DNA recognition by TAL effectors. Science 326:1501

Mundy J, Yamaguchi-Shinozaki K, Chua NH (1990) Nuclear proteins bind conserved elements in the abscisic acid-responsive promoter of a rice rab gene. Proc Natl Acad Sci USA 87:1406-1410

Narusaka Y, Nakashima K, Shinwari ZK, Sakuma Y, Furihata T, Abe H, Narusaka M, Shinozaki K, Yamaguchi-Shinozaki K (2003) Interaction between two cis-acting elements, ABRE and DRE, in ABA-dependent expression of Arabidopsis rd29A gene in response to dehydration and high-salinity stresses. Plant $\mathrm{J}$ 34:137-148

Ohta M, Matsui K, Hiratsu K, Shinshi H, Ohme-Takagi M (2001) Repression domains of class II ERF transcriptional repressors share an essential motif for active repression. Plant Cell 13:1959-1968 
Riechmann JL, Heard J, Martin G, Reuber L, Jiang C, Keddie J, Adam L, Pineda O, Ratcliffe OJ, Samaha RR et al (2000) Arabidopsis transcription factors: genome-wide comparative analysis among eukaryotes. Science 290:2105-2110

Romer P, Hahn S, Jordan T, Strauss T, Bonas U, Lahaye T (2007) Plant pathogen recognition mediated by promoter activation of the pepper Bs3 resistance gene. Science 318:645-648

Szemenyei H, Hannon M, Long JA (2008) TOPLESS mediates auxindependent transcriptional repression during Arabidopsis embryogenesis. Science 319:1384-1386

Tsutsui T, Kato W, Asada Y, Sako K, Sato T, Sonoda Y, Kidokoro S, Yamaguchi-Shinozaki K, Tamaoki M, Arakawa K et al (2009) DEAR1, a transcriptional repressor of DREB protein that mediates plant defense and freezing stress responses in Arabidopsis. J Plant Res 122:633-643

Wang T, Iyer LM, Pancholy R, Shi X, Hall TC (2005) Assessment of penetrance and expressivity of RNAi-mediated silencing of the Arabidopsis phytoene desaturase gene. New Phytol 167:751-760

Weber E, Gruetzner R, Werner S, Engler C, Marillonnet S (2011) Assembly of Designer TAL Effectors by Golden Gate Cloning. PLoS One 6:e19722
Xiong L, Ishitani M, Lee H, Zhu JK (1999a) HOS5-a negative regulator of osmotic stress-induced gene expression in Arabidopsis thaliana. Plant J 19:569-578

Xiong L, Ishitani M, Zhu JK (1999b) Interaction of osmotic stress, temperature, and abscisic acid in the regulation of gene expression in Arabidopsis. Plant Physiol 119:205-212

Yaghmai R, Cutting GR (2002) Optimized regulation of gene expression using artificial transcription factors. Mol Ther 5:685-694

Yamaguchi-Shinozaki K, Shinozaki K (1994) A novel cis-acting element in an Arabidopsis gene is involved in responsiveness to drought, low-temperature, or high-salt stress. Plant Cell 6:251-264

Zhang X, Henriques R, Lin SS, Niu QW, Chua NH (2006) Agrobacterium-mediated transformation of Arabidopsis thaliana using the floral dip method. Nat Protoc 1:641-646

Zhang F, Cong L, Lodato S, Kosuri S, Church GM, Arlotta P (2011) Efficient construction of sequence-specific TAL effectors for modulating mammalian transcription. Nat Biotechnol 29:149-153 